\title{
Effects of Additives on Weather-Resistance Properties of Polyurethane Films Exposed to Ultraviolet Radiation and Ozone Atmosphere
}

\author{
Haiyan Wang, ${ }^{1}$ Yanzhi Wang, ${ }^{1}$ Dongxu Liu, ${ }^{2}$ Zonghui Sun, ${ }^{1}$ and Huicong Wang ${ }^{1}$ \\ ${ }^{1}$ Hebei Key Laboratory of Applied Chemistry, College of Environmental and Chemical Engineering, Yanshan University, \\ Qinhuangdao 066004, China \\ ${ }^{2}$ School of Aeronautic Science and Engineering, Beijing University of Aeronautics and Astronautics, Beijing 10091, China
}

Correspondence should be addressed to Yanzhi Wang; hhwyz@ysu.edu.cn

Received 20 June 2013; Revised 3 December 2013; Accepted 3 December 2013; Published 5 January 2014

Academic Editor: Tifeng Jiao

Copyright (c) 2014 Haiyan Wang et al. This is an open access article distributed under the Creative Commons Attribution License, which permits unrestricted use, distribution, and reproduction in any medium, provided the original work is properly cited.

\begin{abstract}
Three polyurethane films were prepared by adding the antioxidant-1010 and the composite stabilizer to the polyurethane matrix, respectively. The accelerated weathering tests were performed by using self-designed UV/ozone aging test device. The color difference, yellowness index, UV-Vis spectrum, and infrared spectrum were recorded with colorimeter apparatus, UV-Vis spectroscopy, and FT-IR spectroscopy, respectively. The results show that, for the polyurethane film, the composite stabilizer can remarkably decrease UV transmission, the antioxidant-1010 and the composite stabilizer can markedly decrease the photooxidation index and the carbonyl index, respectively, and the antioxidant-1010 can significantly improve the antiyellowing properties after $60 \mathrm{~h}$ exposure. With incremental exposure time for the three films, UV-Vis transmission decreases, the photooxidation index, the carbonyl index, color difference, and yellowness index increase gradually. Under current experimental conditions, the order of $\mathrm{UV} / \mathrm{O}_{3}$ aging resistance from highness to lowness is as follows: the polyurethane film modified by the antioxidant-1010, the polyurethane film modified by composite stabilizer, and the pure polyurethane film.
\end{abstract}

\section{Introduction}

Polyurethane (PU), a polymer containing repeated group $(-\mathrm{NHCOO}-)$ on the main chain, is well-known coating materials in a large variety of commercial and technical applications, including adhesives, sealants, coatings, fibers, thermoplastic parts, elastomers, and both rigid and flexible foams $[1,2]$. PU is available in many formulations and possesses an excellent balance of properties [3]. As an alternative material for the weathering layer of the high-altitude balloon or airship, thermoplastic PU has recently attracted more and more attention, because it has outstanding overall toughness, high tensile strength, tear strength, abrasion resistance properties requiring much less coating weight, low temperature flexibility, fair gas permeability, good handling properties, and good weatherability and ozone resistance properties. Thermoplastic polyurethane can be heat-sealed, adhesively bonded, and laminated to other substrates [4].
However, similar to other polymer materials, exposure of thermoplastic PU to aggressive environments (i.e., UV radiation, thermal exposure, and oxidative atmosphere) causes changes in their physical, chemical, and mechanical characteristics and even loss of use value [5]. Hence, in order to suppress degradation and prolong the service life, the antiaging agent should be added to the polymer. The suitable antiaging agents for PU materials mainly include ultraviolet absorber [6], antioxidant [3], hindered amine [7, 8], and others $[9,10]$.

The UV absorbers include organic and inorganic UV absorbers. Despite the fact that the organic UV absorber has broad applicability, it has the disadvantages of volatility and migration, due to the pure organic compositions, which not only affects the persistent performance but also leads to environment pollution. Inorganic UV absorbers, such as nano- $\mathrm{ZnO}$ and nano- $\mathrm{CeO}_{2}$, have advantages of good chemical stability, thermal stability, nontoxic, odorless, 
wide wavelength range for the UV shielding, and so forth. However, they usually affect the transparency of the film. The advantages of antioxidants-1010 are low costs and good resistance properties, but heat-discoloration resistance is poor. The functions and effects of the different additives are different, and each has its own merits. Excellent performance or synergy effect could be acquired by using composite stabilizers.

In this paper, thermoplastic polyester PU was selected as matrix, and the modified PU films were prepared by adding antioxidants-1010 and composite additives (containing nano$\mathrm{ZnO}$, nano- $\mathrm{CeO}_{2}$, UV-531, UV-327, and the hindered amine622), respectively. The comprehensive UV/ozone aging properties of polyester PU films were studied in a self-designed UV/ozone aging test device. Meanwhile, the effects of antioxidants-1010 and composite additives on the UV/ozone resistance properties of polyester PU film were analyzed, which provide some basis in application of the thermoplastic PU to the weathering layer of high-altitude balloon or airship.

\section{Experiments}

2.1. Raw Materials and Equipment. In this work, the raw materials mainly include the polyester PU pellets (B85A, BASF AG), antioxidants-1010 ( $\geq 98.0 \%$, Nanjing Hualiming Chemical Co., Ltd.), nano-ZnO (20 nm) modified by silane coupling agent (Qinhuangdao Taijihuan Nano Products Co., Ltd.), nano- $\mathrm{CeO}_{2}$ (50 nm, Qinhuangdao Taijihuan Nano Products Co., Ltd.), $\gamma$-methacryloxypropyltrimethoxy silane (KH570, $\geq 98 \%$, Shanghai Huyu Bio Co., Ltd.), UV absorbent of UV-327 ( $\geq 99.0 \%$, Nanjing Hualiming Chemical Co., Ltd.), UV absorbent of UV-531 ( $\geq 99.0 \%$, Shanghai Deyude Co., Ltd.), antioxidant-1010 ( $\geq 98.0 \%$, Nanjing Hualiming Chemical Co., Ltd.), hindered amine-622 ( $\geq 99.0 \%$, Nanjing Hualiming Chemical Co., Ltd.), and N, N-dimethylformamide (DMF) (AR, Tianjin Kaitong Chemical Co., Ltd.). The structures of UV absorbent of UV-327 and UV-531, antioxidant1010, and hindered amine-622 are shown in Scheme 1.

The experiment instruments and equipment mainly include $\mathrm{AD}-\mathrm{XF}-5 \mathrm{P}$ type ozone generating machine (ozone concentrations ranging from 0 to $1000 \mathrm{ppm}$, Jinan Jieanli Technology Co., Ltd.), ACO-318 electromagnetic air compressor (Guangdong Haley Group Co., Ltd.), UVB-313 UV radiation meter (Beijing Normal Photoelectric Instrument Factory), UVB-313EL UV fluorescent tubes (Q-Lab Co., Ltd.), CPR-G6 ozone detection probe (Beijing Shanmeishuimei Environmental Protection Hi-Tech), CR-10 colorimeter (Japan Minolta Co., Ltd.), UV-2550 UV-Vis spectrophotometer (Shimadzu Co., Ltd. Japan), Nicolet IS10 infrared spectrometer (Thermo Fisher Technologies, Inc.), FA2004 electronic balance (Shanghai the Shunyu Hengping Scientific Instrument Co., Ltd.), JFA-II coating machine (Shanghai Environmental Engineering Technology Co., Ltd.), DZF vacuum oven (Shanghai Jinghong Testing Equipment Co., Ltd.), and KQ3200B ultrasonic cleaning (Kunshan Ultrasonic Instrument Co., Ltd.).

2.2. Surface Modification of Nano- $\mathrm{CeO}_{2}$. At room temperature, $5 \mathrm{~g}$ nano- $\mathrm{CeO}_{2}$ and $100 \mathrm{~mL}$ mixed solvent (absolute
TABLE 1: Content of the additives in the films.

\begin{tabular}{lccccc}
\hline \multirow{2}{*}{ Sample } & \multicolumn{5}{c}{ Content of the additives/wt.\% } \\
& PU & UVAI & UVAO & ANT & HALS \\
\hline PU & 100.0 & 0 & 0 & 0 & 0 \\
PU-ANT & 99.5 & 0 & 0 & 0.5 & 0 \\
PU-COM & 96.7 & 2.0 & 0.8 & 0 & 0.5 \\
\hline
\end{tabular}

ethanol: water $=3: 1$ ) were added to a $250 \mathrm{~mL}$ three-necked flask, magnetically stirring for $30 \mathrm{~min}$, and then heated to $85^{\circ} \mathrm{C}$. Silane coupling agent $\mathrm{KH} 570\left(\mathrm{CeO}_{2} / \mathrm{KH}-570=6\right.$ wt. $\left.\%\right)$ was added dropwise with magnetic stirring. After reflux for $4 \mathrm{~h}$ and extraction for $24 \mathrm{~h}$ using Soxhlet extractor, the products were dried in a vacuum oven at $80^{\circ} \mathrm{C}$ for $12 \mathrm{~h}$.

2.3. Preparation of Slurry. PU pellets, inorganic composite ultraviolet absorbent $\left(\mathrm{ZnO}+\mathrm{CeO}_{2}\right)$ (represented as UVAI), composite organic UV absorbent (UV-531 + UV327) (represented as UVAO), antioxidants-1010 (represented as ANT), and hindered amine-622 (represented as HALS) were weighed according to the designed composition (see Table 1) and then added to a mixed solvent in accordance with the volume ratio of $\mathrm{N}, \mathrm{N}$-two dimethylformamide: methyl ethyl ketone: pellets $=7: 2: 1$ with magnetic stirring. After being uniformly dispersed, the pellets were gradually added to the mixed solvent and gradually heated to about $60^{\circ} \mathrm{C}$ and strongly stirred for two hours. The solid content was held to $15 \%$, and the slurry could be obtained.

2.4. Film Preparation. In this work, the films were prepared by two methods. For the first method, the film was coated on a coating machine and then dried in a vacuum oven at $60^{\circ} \mathrm{C}$ for $12 \mathrm{~h}$. The slurry was cured on the quartz slide, and the film with theoretical thickness of $30 \mu \mathrm{m}$ was obtained for testing the color difference, the yellowness index, and the UV-Vis transmittance. For the other method, the film was prepared by spin-coating with the ratio of $2000 \mathrm{r} / \mathrm{min}$ and the duration of $10 \mathrm{~s}$. After being dried in a vacuum oven at $60^{\circ} \mathrm{C}$ for $12 \mathrm{~h}$, the slurry was cured on $\mathrm{CaF}_{2}$ slide. The obtained film is used for testing the infrared spectra.

2.5. Weatherability Testing. The integrated aging properties of the polyester films were studied in a self-designed comprehensive UV/ozone aging test device. The light source is fluorescent UV lamps of UVB-313 EL system from American Q-Lab Co.; the intensity of ultraviolet irradiation was $400 \pm$ $20 \mu \mathrm{W} / \mathrm{cm}^{2}$ at wavelength of $313 \mathrm{~nm}$. The ozone concentration was $100 \pm 2 \mathrm{ppm}$ at ambient temperature and relative humidity of $20 \%$.

The color difference, the yellowness index, UV-Vis transmittance, and FT-IR spectroscopy of the samples after exposure time of $0 \mathrm{~h}, 20 \mathrm{~h}, 60 \mathrm{~h}, 120 \mathrm{~h}, 160 \mathrm{~h}$, and $200 \mathrm{~h}$ were tested by using CR-10 type colorimeter, UV-2550 UV-Vis spectrophotometer, and Nicolet IS10 infrared spectrometer, respectively.

In order to quantitatively compare the degree of aging, $-\mathrm{CH}$ peak was as internal standard peak. The photooxidation 
UV absorbent of UV-327<smiles>CC(C)(C)c1cc(-n2nc3ccc(Cl)cc3n2)c(O)c(C(C)(C)C)c1</smiles>

UV absorbent of UV-531

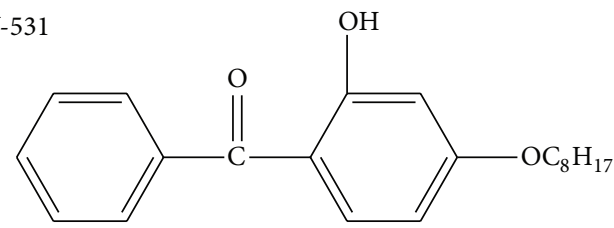

Antioxidant-1010<smiles>CCOC(=O)CCc1cc(C(C)(C)C)c(O)c(C(C)(C)C)c1</smiles>

Hindered amine-622<smiles>COC(=O)CCC(=O)OCCN1C(C)(C)CC(C)(C)CC1(C)C</smiles>

Scheme 1: Structures of UV absorbent of UV-327 and UV-531, antioxidant-1010, and hindered amine-622.

index (PI) and the carbonyl index (CI) were calculated according to formulas (1) and (2), respectively:

$$
\begin{gathered}
P I=\left(\frac{A}{B}\right)_{\text {aged }}-\left(\frac{A}{B}\right)_{\text {initial }} \\
C I=\frac{C}{B},
\end{gathered}
$$

wherein $A$ represents $-\mathrm{OH}$ and $-\mathrm{NH}$ peak area and $B$ and $C$ represent the peak areas of $-\mathrm{CH}_{3}$ and $-\mathrm{C}=\mathrm{O}$, respectively.

\section{Results and Discussion}

3.1. UV-Vis Spectra. UV-Vis transmittance spectra of the PU, PU-ANT, and PU-COM films as function of the aging time are shown in Figures 1 and 2. It is found that UV transmittance of the film gradually decreases as the aging time increases before $120 \mathrm{~h}$, indicating that the aging degree of the film increases with the increase of the aging time. After $120 \mathrm{~h}$, UV transmittance of the film remains stable. However, as can be seen from Figure 2, UV transmittance of the PUCOM film is significantly lower than those of PU and PUANT films during the same aging time, indicating that the composite additive could significantly improve the UV/ozone resistance properties of the polyester PU material. This may be attributed to the composite UV absorbent which could effectively shield or absorb ultraviolet light and thus inhibit or reduce the rate of oxidation reaction of the incremental macromolecular and postpone the degradation and aging process of the PU material. 


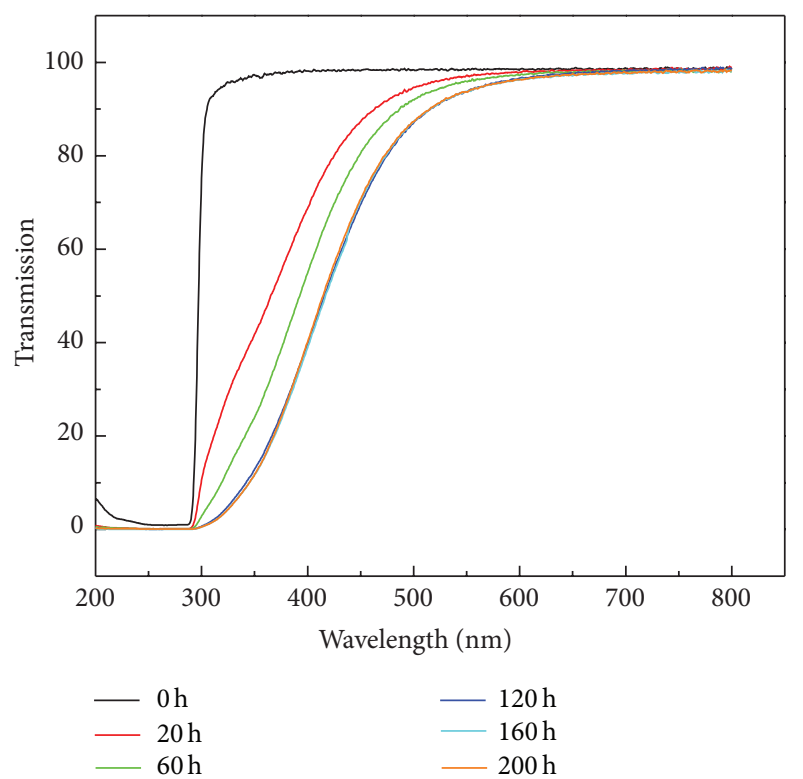

FIGURE 1: UV-Vis transmittance spectra of PU film with the increase of the aging time.

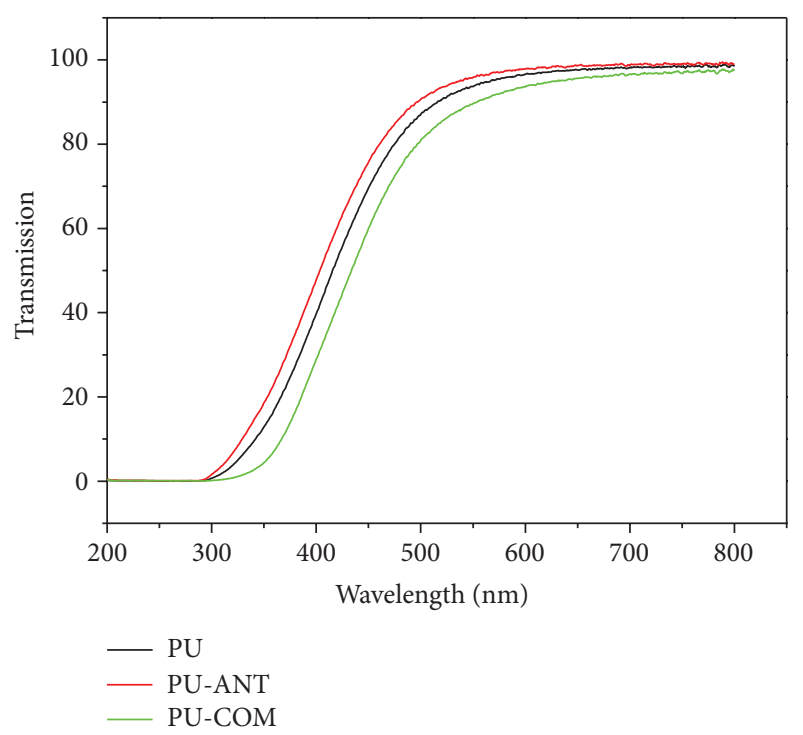

FIgURe 2: UV-Vis transmittance spectra of PU, PU-ANT, and PUCOM films at the aging time of $120 \mathrm{~h}$.

3.2. FT-IR Spectra. FT-IR spectra of the PU, PU-ANT, and PU-COM films as function of the aging time are shown in Figures 3, 4, and 5. The following main peaks can be observed from the spectra: $3500 \sim 3000 \mathrm{~cm}^{-1}:-\mathrm{NH} / \mathrm{OH}$ stretching vibration peak; $3000 \sim 2800 \mathrm{~cm}^{-1}$ : $-\mathrm{CH}_{2}$ asymmetric and symmetric stretching peak; $1760 \sim 1660 \mathrm{~cm}^{-1}$ : $-\mathrm{C}=\mathrm{O}$ stretching vibration peak; $1527 \mathrm{~cm}^{-1}$ : the coupling peak of the $-\mathrm{NH} / \mathrm{OH}$ bending vibration and $\mathrm{C}-\mathrm{N}$ stretching vibration in $-\mathrm{C}-\mathrm{NH}$ group; $1300 \sim 1100 \mathrm{~cm}^{-1}$ : the $\mathrm{C}-\mathrm{O}$ stretching vibration in ester group.

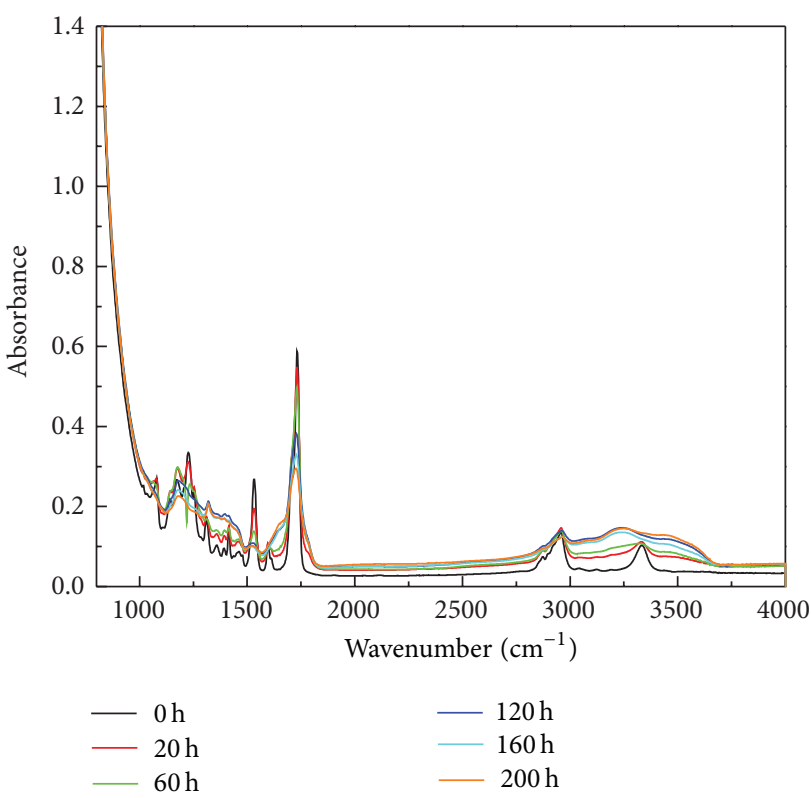

FIGURE 3: FT-IR spectra of PU film as function of the aging time.

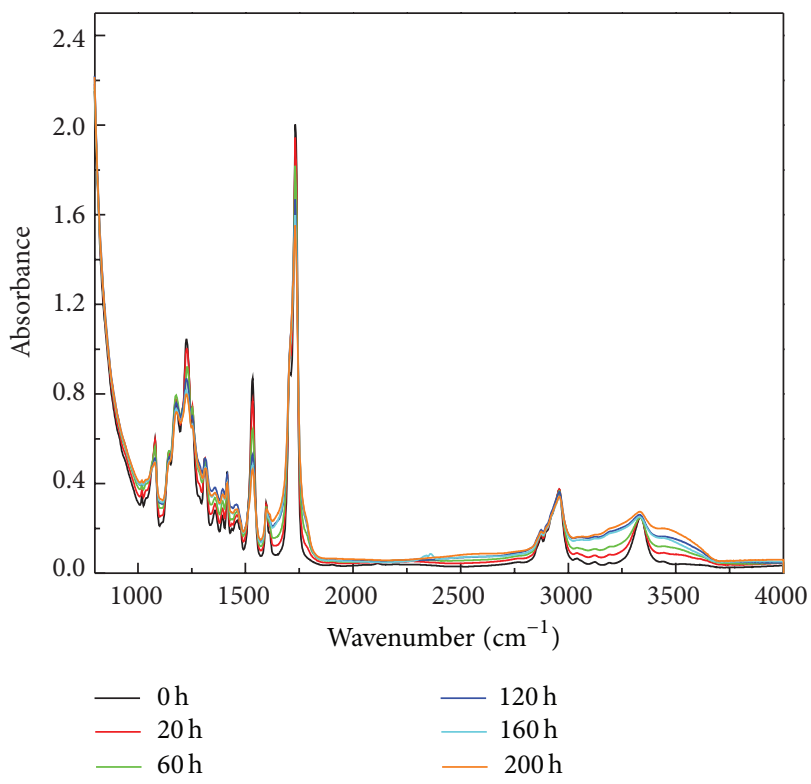

FIGURE 4: FT-IR spectra of PU-ANT film as function of the aging time.

It can also be seen from Figure 3 to Figure 5 that the peak of $-\mathrm{NH} / \mathrm{OH}$ stretching vibration widens and the peak area increases gradually with the increase of the aging time, indicating that the hydrogen peroxide may be generated during the $\mathrm{UV} / \mathrm{O}_{3}$ aging process [11]. The $-\mathrm{C}=\mathrm{O}$ peak widens gradually with the increase of the aging time, indicating that new carbonyl groups (including quinone-imide structure) may be generated during the $\mathrm{UV} / \mathrm{O}_{3}$ aging process [7]. These changes show that the urethane structures of these films gradually decrease as the aging time increases. In addition, the peak height at $1527 \mathrm{~cm}^{-1}$ in the spectra gradually 


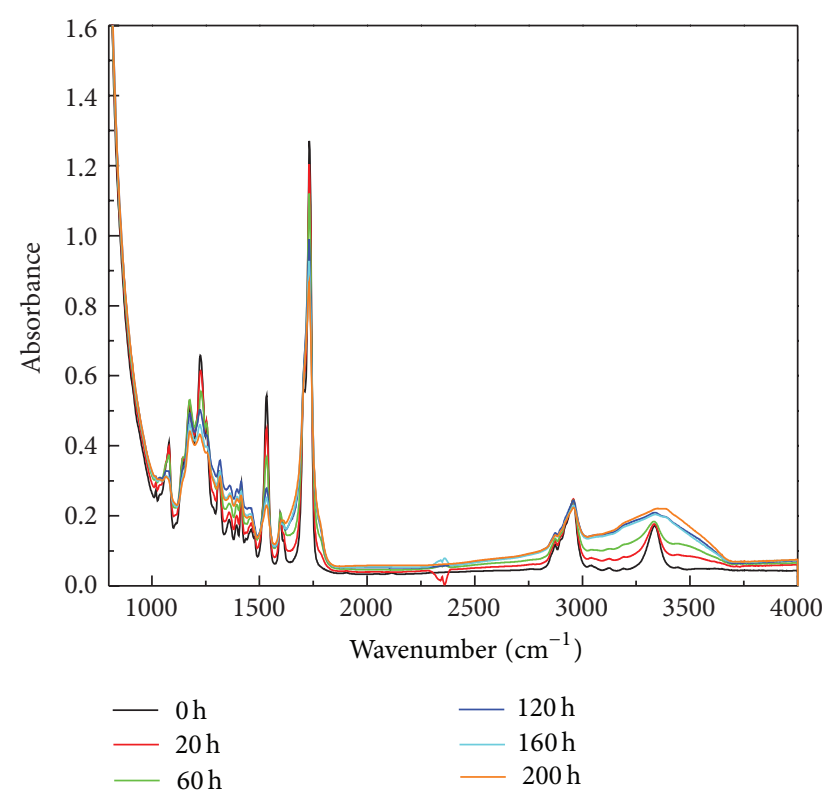

FIGURE 5: FT-IR spectra of PU-COM film as function of the aging time.

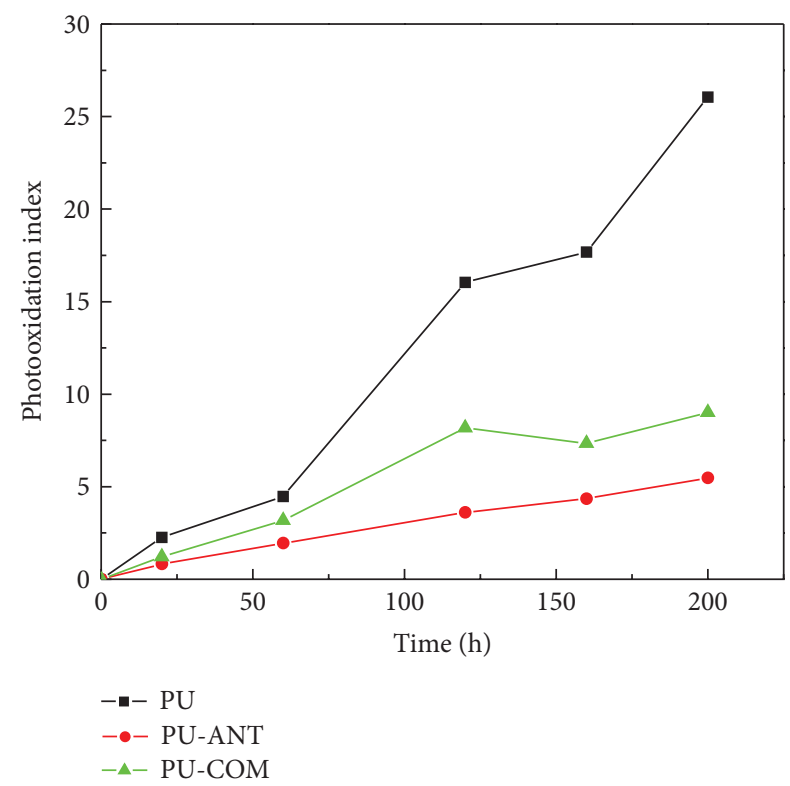

FIgUre 6: The variation of PI of PU, PU-ANT, and PU-COM films as function of the aging time.

decreases with the increase of the aging time, which indicates that the Photo-Fries rearrangement of the urethane structure may occur in each film [2].

3.3. The Photooxidation Index (PI) and the Carbonyl Index (CI). In order to quantitatively compare the aging degrees of PU, PU-ANT, and PU-COM films during the same aging time, $-\mathrm{CH}$ peak was as internal standard peak. The photooxidation index (PI) and the carbonyl index (CI) were calculated according to formulas (1) and (2), and the results

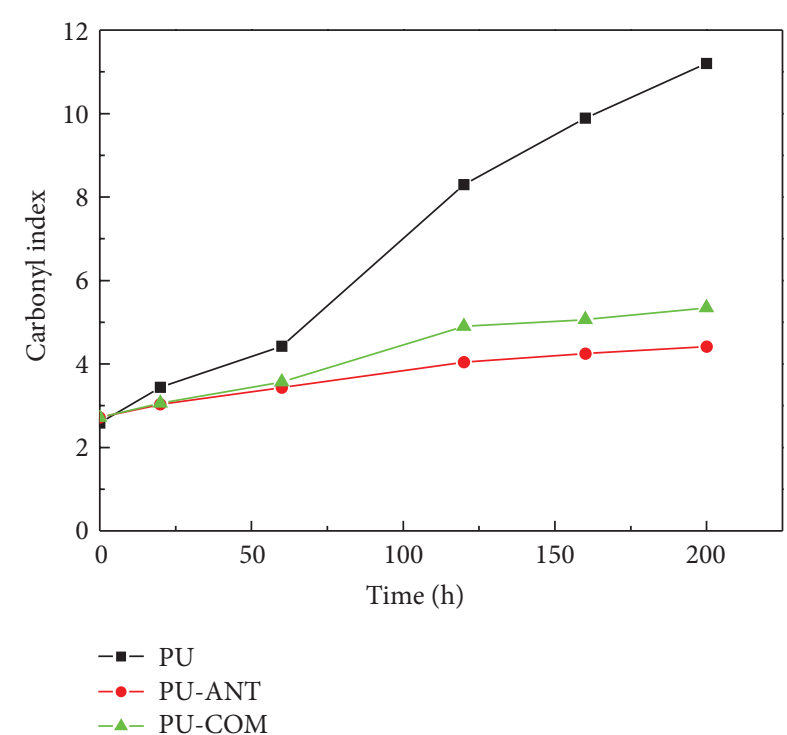

FIgURE 7: The variation of CI of PU, PU-ANT, and PU-COM films as function of the aging time.

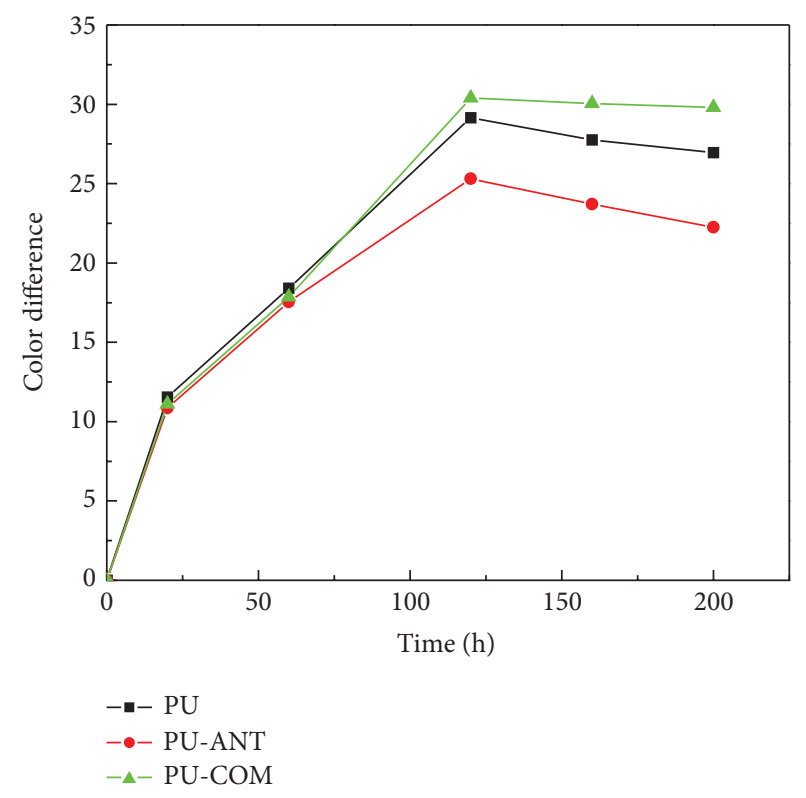

FIGURE 8: The variation of the color difference of PU, PU-ANT, and $\mathrm{PU}-\mathrm{COM}$ films as function of the aging time.

are shown in Figures 6 and 7, respectively. It can be seen that both the photooxidation index and the carbonyl index of the films gradually increase with the increase of the aging time, because the ozone has strong oxidation property and the ultraviolet light has enough energy for penetrating, which accelerates radical generation and enhances the photoradical reaction. However, when the aging time is more than $60 \mathrm{~h}$, the photooxidation index and the carbonyl index of the PU film increase rapidly with the increase of the aging time, while those of modified PU-ANT and PU-COM films increase very slowly, especially the PU-ANT film. For example, at the aging 


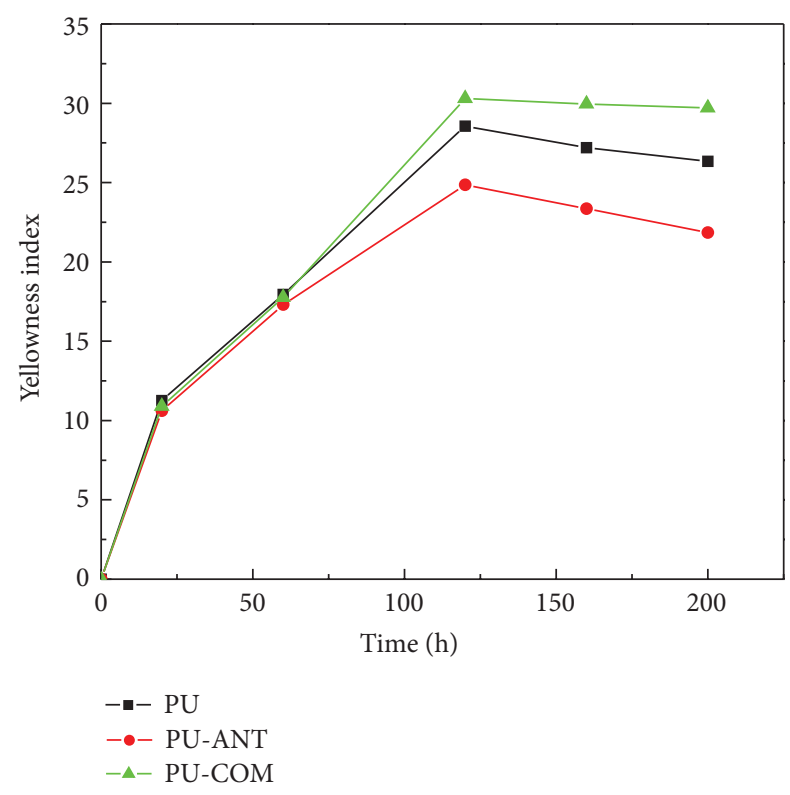

FIGURE 9: The variation of the yellowness index of PU, PU-ANT, and PU-COM films as function of the aging time.

time of $200 \mathrm{~h}$, photooxidation index of PU-COM film is 9.01, which is lower than that of PU film (26.05) significantly, because the composite additive could effectively shield and absorb UV as well as hinder the aging of PU. PU-ANT film has the lowest photooxidation index of 5.47, indicating that $0.5 \mathrm{wt} \%$ antioxidant-1010 could significantly delay the degradation and the aging process of PU in the UV/ozone environment. Moreover, the composition and the ratio of the composite additive could be further optimized.

3.4. The Color Difference and the Yellowness Index. The color difference and yellowness index of PU, PU-ANT, and PU$\mathrm{COM}$ films as function of the aging time are shown in Figures 8 and 9, respectively. It can be seen from Figure 8 that the variation of the color difference of the modified PU is similar to that of pure PU. Before $120 \mathrm{~h}$, as the aging time increases, the color difference of each film increases without significant difference from each other. Thereafter, the color difference of each film is nearly unchanged. However, the color difference of PU-ANT film is significantly lower than that of PU film, while the color difference of PU-COM film is higher than that of PU film. As can be seen from Figure 9, the variation of yellowness index for each film is basically consistent with that of the color difference. These phenomena indicate that in the UV/ozone environment, the methylene in PU film may be oxidized, leading to the quinone-imide structure chromophore [6]. With the increase of the aging time, the yellowing resistance properties of PU-ANT film are much better than those of the pure PU, indicating that antioxidant-1010 can effectively improve the yellowing resistance properties of $\mathrm{PU}$ film. A possible reason is that the antioxidant can effectively suppress or reduce the rate of thermal oxidation and photooxidation of macromolecules, thereby significantly improve the heat resistance and the light resistance properties of the material and delay the degradation of the $\mathrm{PU}$ material during the aging process. After $120 \mathrm{~h}$, the yellowing resistance properties of PU-COM film are slightly lower than those of the pure PU, which could be attributed to the decomposition and failure of some organic additives in the strong UV/ozone environment or certain antagonism effect among some additives. Further studies need to be performed for verifying the exact reason.

\section{Conclusions}

(1) The composite stabilizer can significantly reduce the UV transmittance of the PU film. The UV transmittances of three films gradually decrease with the increase of the aging time.

(2) The $-\mathrm{NH} / \mathrm{OH}$ and $-\mathrm{C}=\mathrm{O}$ peaks of three films gradually widen, and the urethane structures gradually reduce with the increase of the aging time.

(3) Antioxidant-1010 and the composite stabilizer are able to significantly reduce photooxidation index and the carbonyl index of the polyester PU film. In the present experimental conditions, the order of $\mathrm{UV} / \mathrm{O}_{3}$ aging resistance properties of these films is PU-ANT, PU$\mathrm{COM}$, and PU film.

(4) The color difference and the yellowness index of these films gradually increase with the increase of the aging time. In the early stage of aging, the variations of the color difference and the yellowness index are not obvious. As the aging time is more than $60 \mathrm{~h}$, the yellowing resistance properties of PU-ANT film are better than those of PU-COM and PU films.

\section{Conflict of Interests}

The authors declare that there is no conflict of interests regarding the publication of this paper.

\section{References}

[1] A. Boubakri, N. Guermazi, K. Elleuch, and H. F. Ayedi, "Study of UV-aging of thermoplastic polyurethane material," Materials Science and Engineering A, vol. 527, no. 7-8, pp. 1649-1654, 2010.

[2] D. Rosu, L. Rosu, and C. N. Cascaval, "IR-change and yellowing of polyurethane as a result of UV irradiation," Polymer Degradation and Stability, vol. 94, no. 4, pp. 591-596, 2009.

[3] R. P. Singh, N. S. Tomer, and S. V. Bhadraiah, "Photo-oxidation studies on polyurethane coating: effect of additives on yellowing of polyurethane," Polymer Degradation and Stability, vol. 73, no. 3, pp. 443-446, 2001.

[4] H. Zhai and A. Euler, "Material challenges for lighter-thanair systems in high altitude applications," in Proceedings of the AIAA 5th Aviation Technology, Integration, and Operations (ATIO) Conference: AIAA 16th Lighter-than-Air Systems Technology Conference and Balloon Systems Conference, S. Danielle, Ed., pp. 26-28, American Institute of Aeronautics and Astronautics, Virginia, Va, USA, 2005.

[5] A. Boubakri, N. Haddar, K. Elleuch, and Y. Bienvenu, "Impact of aging conditions on mechanical properties of thermoplastic 
polyurethane," Materials and Design, vol. 31, no. 9, pp. 41944201, 2010.

[6] M. Rashvand, Z. Ranjbar, and S. Rastegar, "Nano zinc oxide as a UV-stabilizer for aromatic polyurethane coatings," Progress in Organic Coatings, vol. 71, no. 4, pp. 362-368, 2011.

[7] M. E. Nichols and J. L. Gerlock, "Rates of photooxidation induced crosslinking and chain scission in thermoset polymer coatings. II. Effect of hindered amine light stabilizer and ultraviolet light absorber additives," Polymer Degradation and Stability, vol. 69, no. 2, pp. 197-207, 2000.

[8] H. Jia, H. Wang, and W. Chen, "The combination effect of hindered amine light stabilizers with UV absorbers on the radiation resistance of polypropylene," Radiation Physics and Chemistry, vol. 76, no. 7, pp. 1179-1188, 2007.

[9] J. Salla, K. K. Pandey, and K. Srinivas, "Improvement of UV resistance of wood surfaces by using $\mathrm{ZnO}$ nanoparticles," Polymer Degradation and Stability, vol. 97, no. 4, pp. 592-596, 2012.

[10] A. Saadat-Monfared, M. Mohseni, and M. Hashemi Tabatabaei, "Polyurethane nanocomposite films containing nanocerium oxide as UV absorber, part 1: static and dynamic light scattering, small angle neutron scattering and optical studies," Colloids and Surfaces A, vol. 408, pp. 64-70, 2012.

[11] C. S. Schollenberger and F. D. Stewart, "Thermoplastic urethane structure and ultraviolet stability," Journal of Elastomers and Plastics, vol. 4, no. 4, pp. 294-331, 1972. 

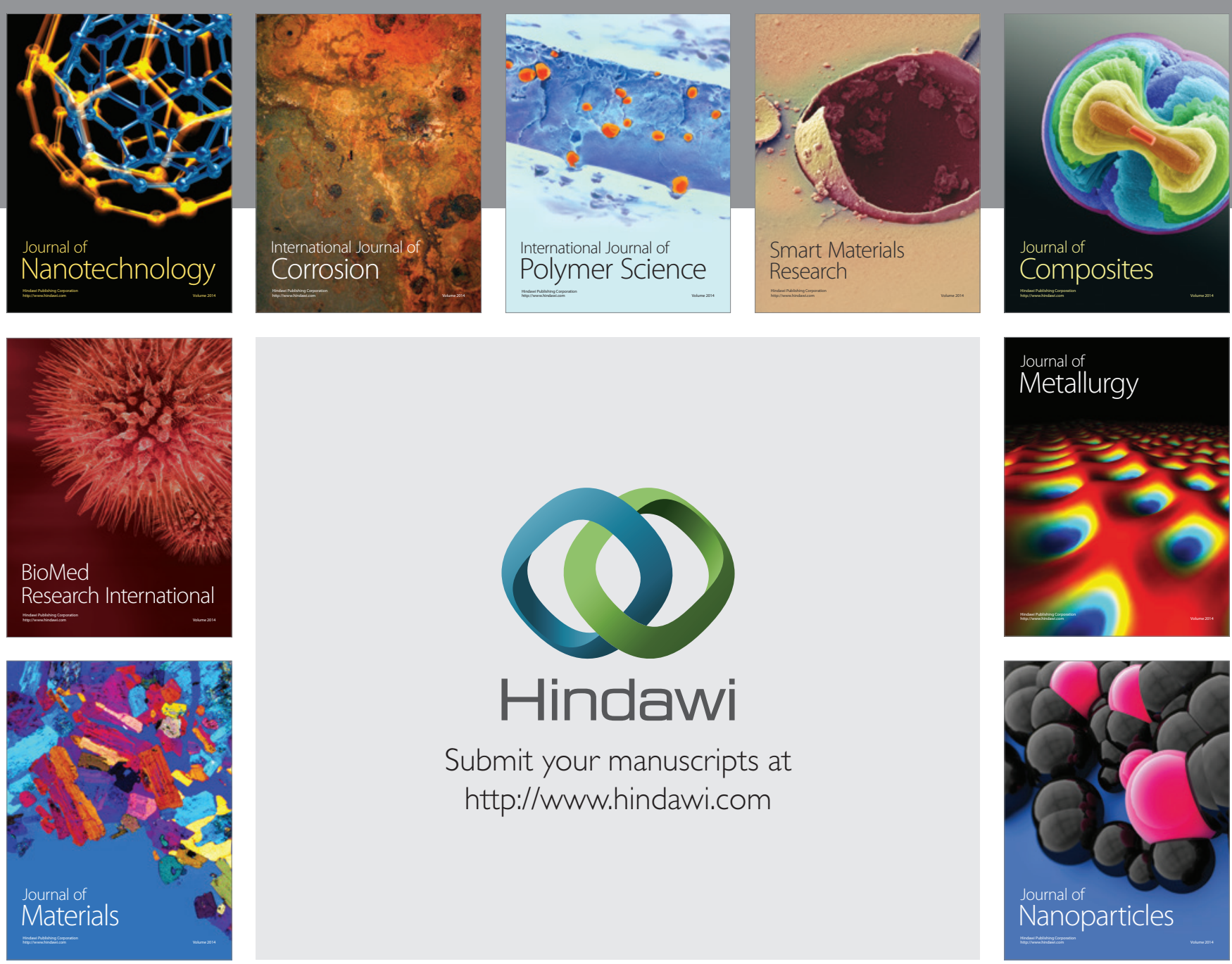

Submit your manuscripts at http://www.hindawi.com
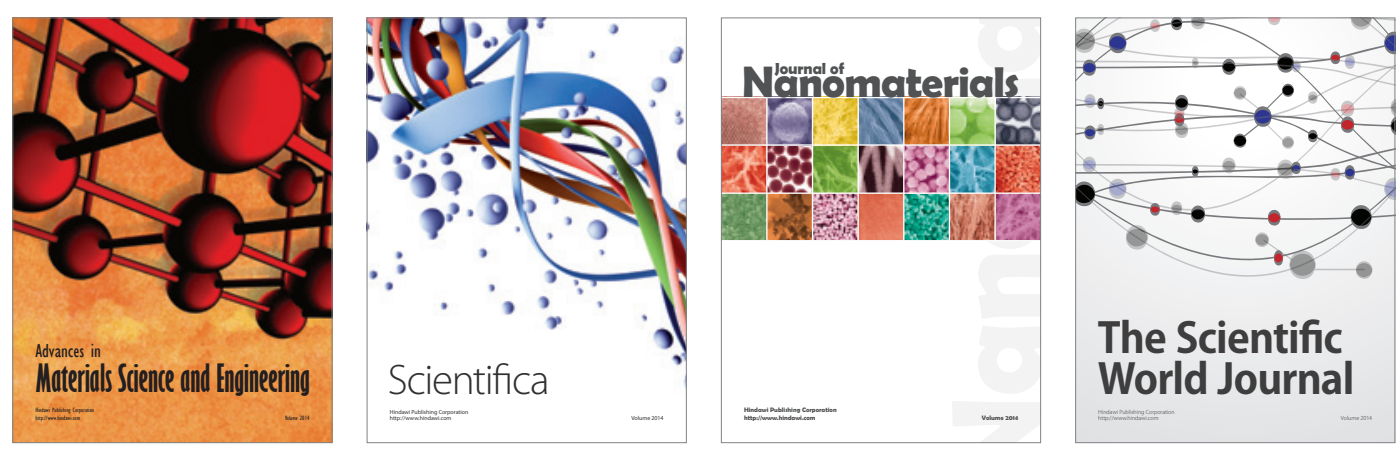

\section{The Scientific World Journal}
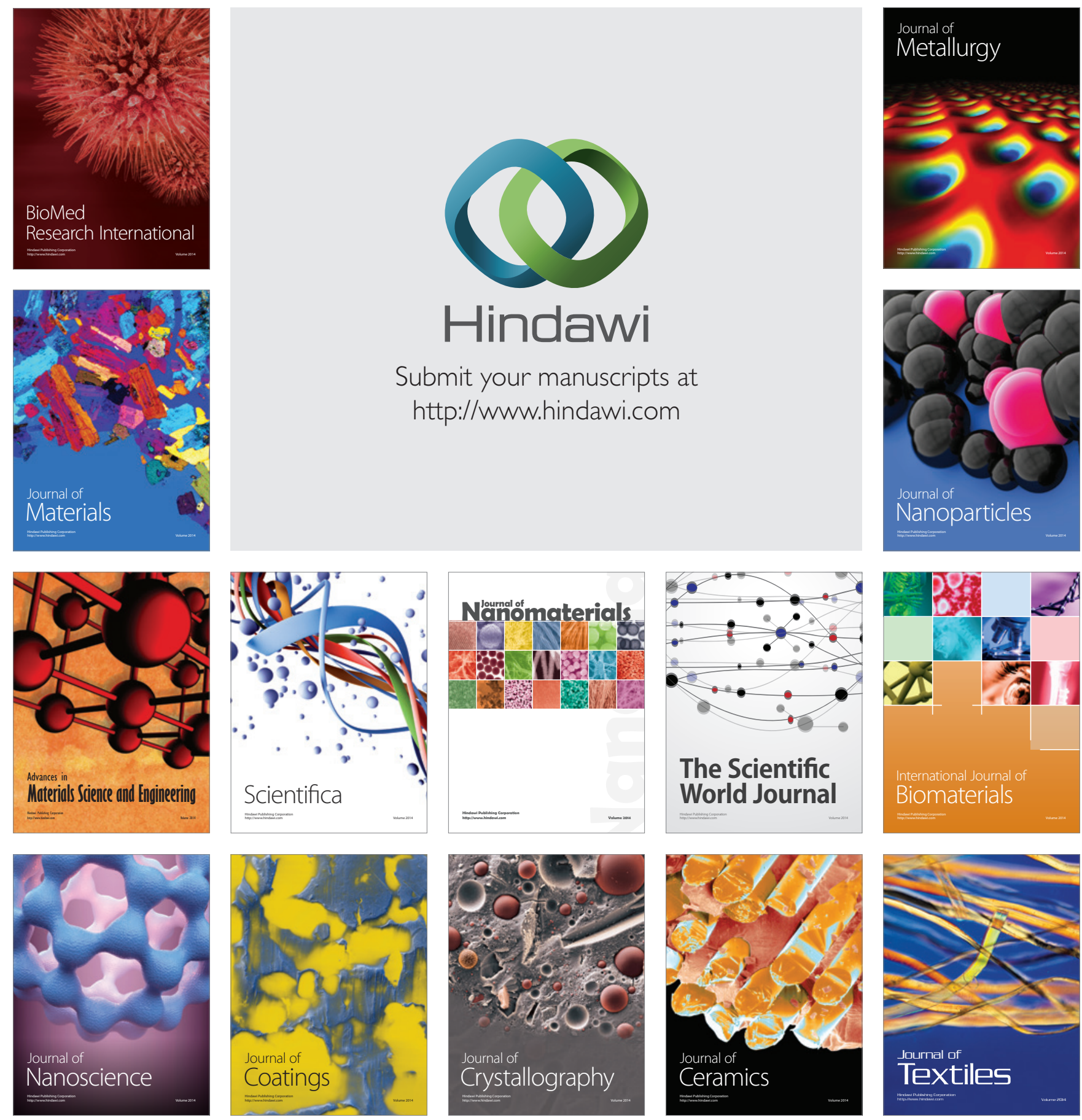